\title{
Novel Antimicrobial Agents: Fluorinated 2-(3-(Benzofuran-2-yl) pyrazol-1-yl)thiazoles
}

\author{
Hanan A. Mohamed, ${ }^{1}$ Ehab Abdel-Latif, ${ }^{2}$ Bakr F. Abdel-Wahab, ${ }^{1}$ and Ghada E. A. Awad ${ }^{3}$ \\ ${ }^{1}$ Applied Organic Chemistry Department, National Research Centre, Dokki, Giza 12622, Egypt \\ ${ }^{2}$ Department of Chemistry, Faculty of Science, Mansoura University, Mansoura 35516, Egypt \\ ${ }^{3}$ Chemistry of Natural and Microbial Products, National Research Center, Dokki, Giza 12622, Egypt
}

Correspondence should be addressed to Bakr F. Abdel-Wahab; balshobia@su.edu.sa

Received 29 May 2013; Revised 10 August 2013; Accepted 11 August 2013

Academic Editor: Armando Rossello

Copyright (C) 2013 Hanan A. Mohamed et al. This is an open access article distributed under the Creative Commons Attribution License, which permits unrestricted use, distribution, and reproduction in any medium, provided the original work is properly cited.

A new series of 2-pyrazolin-1-ylthiazoles 8a-d and 13-16 was synthesized by cyclization of $N$-thiocarboxamide-2-pyrazoline with different haloketones and 2,3-dichloroquinoxaline. The structures of the new compounds were confirmed by elemental analyses as well as NMR, IR, and mass spectral data. The newly synthesized compounds were evaluated for their antimicrobial activities, and also their minimum inhibitory concentration (MIC) against most of test organisms was performed. Amongst the tested ones, compound $\mathbf{8 c}$ displayed excellent antimicrobial activity.

\section{Introduction}

Pyrazolines are nitrogen-containing heterocyclic compounds, well known for their pronounced biological activity. These biological activities include antibacterial [1], antifungal [2], herbicidal [3], and insecticidal activities [4]. It was demonstrated that the combination of pyrazole with azole ring, linked to each by one sigma bond, led to more biologically active targets; for example, pyrazolylthiazoles showed excellent antimicrobial activities [5]. Continuing our work in this research field [6-9] and in an attempt to identify new and potent antimicrobial agents, we tried here to generate new benzofuryl 2-pyrazolin-1-ylthiazoles as antimicrobial agents using simple methods.

\section{Results and Discussion}

2.1. Chemistry. The starting pyrazoline-1-carbothioamide $\mathbf{5}$ was prepared by treatment of 2-acetylbenzofuran 1 with equivalent of 4-flurobenzaldehyde 2 in the presence of $10 \%$ alcoholic $\mathrm{NaOH}$ in $90 \%$ ethanol with stirring at room temperature to give chaconne 3 . Reaction of chalcone 3 with equivalent amount of thiosemicarbazide was performed in ethanol in the presence of 2.5 equivalent of sodium hydroxide to the target precursor 5 .
The resulting pyrazoline-1-carbothioamide 5 was cyclized to the corresponding 2-(3-(benzofuran-2-yl)-5-(4fluorophenyl)-4,5-dihydro-1H-pyrazol-1-yl)-4-methyl-5-( $p$ subs.phenyldiazenyl)thiazole derivatives $\mathbf{8 a}-\mathbf{d}$ by reaction with hydrazonoyl halides $\mathbf{6 a - d}$ in anhydrous ethanol and in the presence of an equivalent of triethylamine (Scheme 1).

The reaction product structures were elucidated by means of NMR, MS spectroscopy, and elemental analyses (Table 1).

For example, ${ }^{1} \mathrm{H}$ NMR spectra of $\mathbf{8 a}-\mathbf{d}$ contained two doublet-doublet and one triplet signals due to the presence of $\mathrm{CH}_{2}$ adjacent asymmetric carbon. The mass spectra of $\mathbf{8 a}-\mathbf{d}$ showed the molecular ion peaks at $m / z 481,499,515$ and 561, respectively in agreement with the calculated masses.

The reaction between pyrazoline-1-carbothioamide 5 and the equivalent amount of $\alpha$-haloketones, for example, phenacyl bromides derivatives, 2-bromoacetylbenzofuran, and 3bromoacetylcoumarin, was performed in refluxing ethanol to yield pyrazol-1-ylthiazoles 13-15 in good yields via the intermediates A (Scheme 2). Also, pyrazoline-1-carbothioamide 5 reacted with 2,3-dichloroquinoxaline to give pyrazolin-1ylthiazolo[5,4-b]quinoxaline $\mathbf{1 6}$ in moderate yield.

The ${ }^{1} \mathrm{H}$ NMR of compounds 13-16 indicate the disappearance of $\mathrm{NH}_{2}$ signal due to blocking of $\mathrm{NH}_{2}$ with thiazole nucleus. The mass spectra of $\mathbf{1 3} \mathbf{a}, \mathbf{b}-\mathbf{1 6}$ showed the molecular 
<smiles>CC(=O)c1cc2ccccc2o1</smiles>

1<smiles>O=Cc1ccc(F)cc1</smiles>

$\underset{-\mathrm{H}_{2} \mathrm{O}}{\stackrel{10 \% \text { Alc. } \mathrm{NaOH}}{\longrightarrow}}$<smiles>O=C(/C=C/c1ccc(F)cc1)c1cc2ccccc2o1</smiles>

3<smiles>[R]C(=O)/C(Cl)=N\Nc1ccc([R])cc1</smiles>
$\mathrm{EtOH} / \mathrm{Et}_{3} \mathrm{~N}$ $-\mathrm{HCl}$

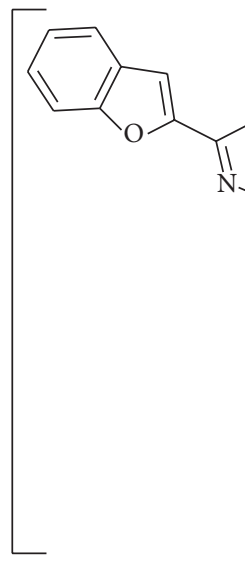<smiles>NC(=S)N1N=C(c2cc3ccccc3o2)CC1c1ccc(F)cc1</smiles>

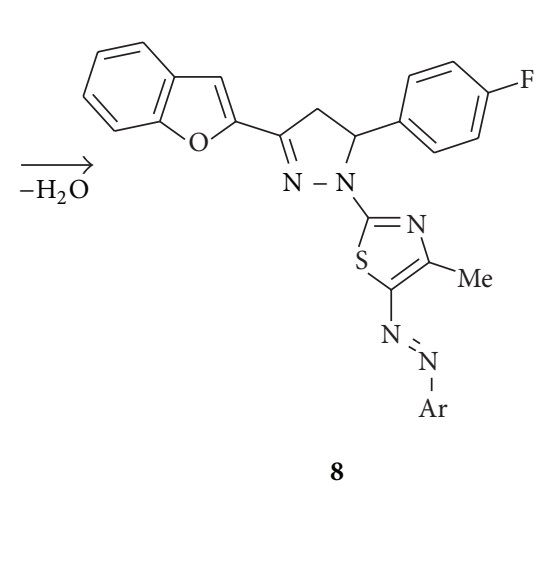

$\mathrm{Ar}=\mathrm{Ph}, 4-\mathrm{FC}_{6} \mathrm{H} 4,4-\mathrm{CIC}_{6} \mathrm{H}_{4}, 4-\mathrm{BrC}_{6} \mathrm{H}_{4}$

SCHEME 1

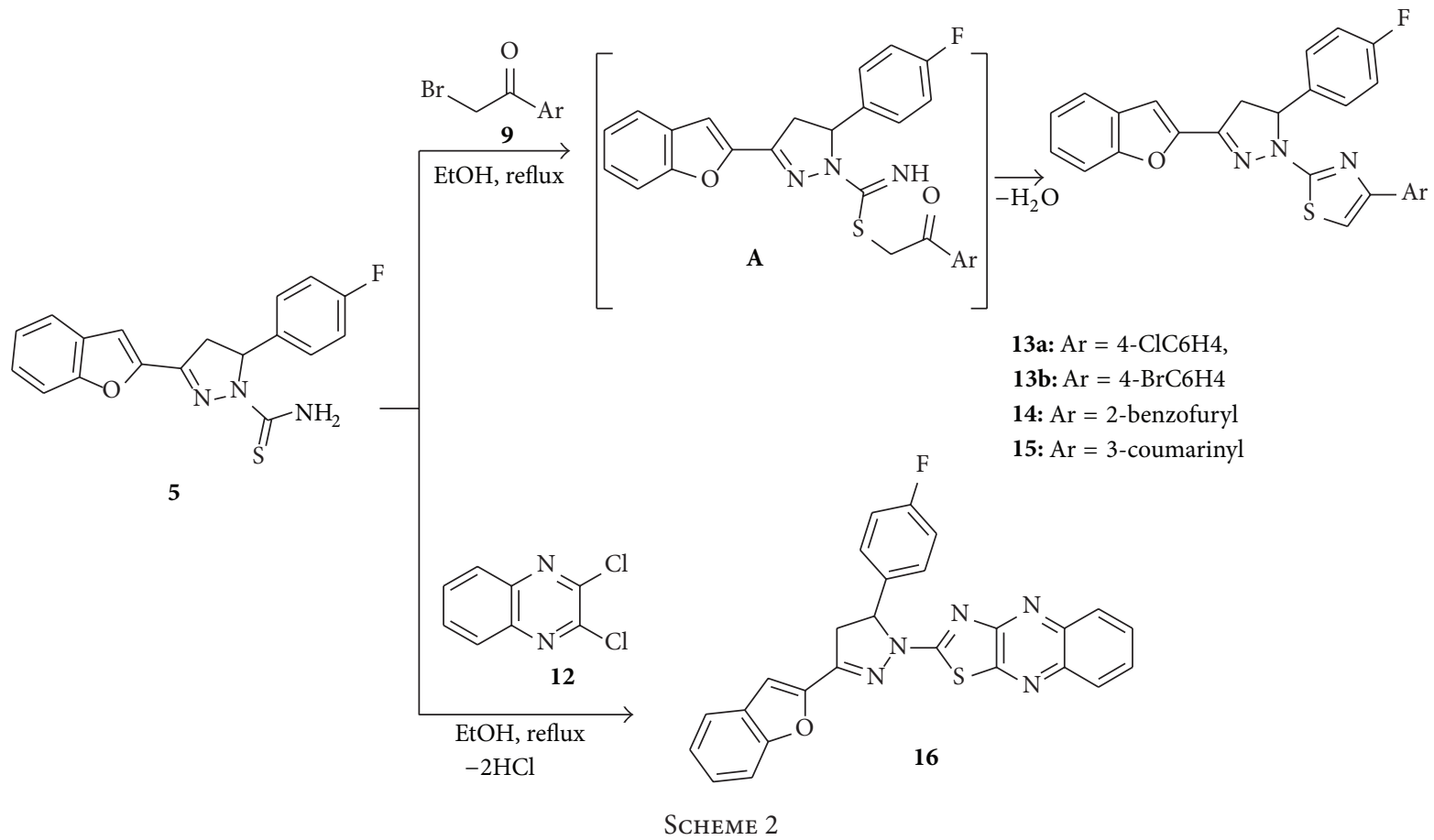


TABLE 1: Characteristic data of the synthesized compounds.

\begin{tabular}{lcccc}
\hline & & & Calcd. & \\
Entry & Mol. formula & & Found & \\
& $(\mathrm{M} . \mathrm{Wt})$ & $\mathrm{C} \%$ & $\mathrm{H} \%$ & $\mathrm{~N} \%$ \\
\hline \multirow{2}{*}{$\mathbf{5}$} & $\mathrm{C}_{18} \mathrm{H}_{14} \mathrm{FN}_{3} \mathrm{OS}$ & $\mathbf{6 3 . 7 0}$ & $\mathbf{4 . 1 6}$ & $\mathbf{1 2 . 3 8}$ \\
& $(339.39)$ & 64.03 & 4.20 & 12.51 \\
$\mathbf{8 a}$ & $\mathrm{C}_{27} \mathrm{H}_{20} \mathrm{FN}_{5} \mathrm{OS}$ & $\mathbf{6 7 . 3 4}$ & $\mathbf{4 . 1 9}$ & $\mathbf{1 4 . 5 4}$ \\
& $(481.54)$ & 67.54 & 4.31 & 14.35 \\
$\mathbf{8 b}$ & $\mathrm{C}_{27} \mathrm{H}_{19} \mathrm{~F}_{2} \mathrm{~N}_{5} \mathrm{OS}$ & $\mathbf{6 4 . 9 2}$ & $\mathbf{3 . 8 3}$ & $\mathbf{1 4 . 0 2}$ \\
& $(499.53)$ & 64.98 & 3.90 & 14.22 \\
$\mathbf{8 c}$ & $\mathrm{C}_{27} \mathrm{H}_{19} \mathrm{ClFN}_{5} \mathrm{OS}$ & $\mathbf{6 2 . 8 5}$ & $\mathbf{3 . 7 1}$ & $\mathbf{1 3 . 5 7}$ \\
& $(515.99)$ & 62.94 & 3.83 & 13.66 \\
$\mathbf{8 d}$ & $\mathrm{C}_{27} \mathrm{H}_{19} \mathrm{BrFN}_{5} \mathrm{OS}$ & $\mathbf{5 7 . 8 6}$ & $\mathbf{3 . 4 2}$ & $\mathbf{1 2 . 5 0}$ \\
& $(560.44)$ & 57.89 & 3.39 & 12.59 \\
$\mathbf{1 3 a}$ & $\mathrm{C}_{26} \mathrm{H}_{17} \mathrm{ClFN}_{3} \mathrm{OS}$ & $\mathbf{6 5 . 8 9}$ & $\mathbf{3 . 6 2}$ & $\mathbf{8 . 8 7}$ \\
& $(473.95)$ & 65.93 & 3.59 & 8.93 \\
$\mathbf{1 3 b}$ & $\mathrm{C}_{26} \mathrm{H}_{17} \mathrm{BrFN}_{3} \mathrm{OS}$ & $\mathbf{6 0 . 2 4}$ & $\mathbf{3 . 3 1}$ & $\mathbf{8 . 1 1}$ \\
& $(518.40)$ & 60.30 & 3.39 & 8.31 \\
$\mathbf{1 4}$ & $\mathrm{C}_{28} \mathrm{H}_{18} \mathrm{FN}_{3} \mathrm{O}_{2} \mathrm{~S}$ & $\mathbf{7 0 . 1 3}$ & $\mathbf{3 . 7 8}$ & $\mathbf{8 . 7 6}$ \\
& $(479.52)$ & 70.26 & 3.82 & 8.89 \\
& $\mathrm{C}_{29} \mathrm{H}_{18} \mathrm{FN}_{3} \mathrm{O}_{3} \mathrm{~S}$ & $\mathbf{6 8 . 6 3}$ & $\mathbf{3 . 5 7}$ & $\mathbf{8 . 2 8}$ \\
$\mathbf{1 5}$ & $(507.53)$ & 68.77 & 3.66 & 8.33 \\
& $\mathrm{C}_{26} \mathrm{H}_{16} \mathrm{FN}_{5} \mathrm{OS}$ & $\mathbf{6 7 . 0 8}$ & $\mathbf{3 . 4 6}$ & $\mathbf{1 5 . 0 4}$ \\
$\mathbf{1 6}$ & $(465.50)$ & 67.14 & 3.50 & 15.11 \\
\hline & & & & \\
& & & &
\end{tabular}

ion peaks at $m / z 473,518,479,507$, and 465 , respectively in agreement with the calculated masses.

\subsection{Biological Activity}

2.2.1. Antimicrobial Activity. All the new synthesized compounds were screened for their antibacterial and antifungal activities at $100 \mu \mathrm{g} / \mathrm{mL}$ concentration against four Grampositive bacteria (Staphylococcus aureus ATCC 29213; B. subtilis ATCC6633; B. megaterium ATCC 9885; Sarcina lutea), three Gram-negative bacteria (Klebsiella pneumoniae ATCC13883; Pseudomonas aeruginosa ATCC27953; E. coli ATCC 25922), and two yeast (Saccharomyces cerevisiae and Candida albicans NRRL Y-477). Ciprofloxacin and ketoconazole were respectively used as standard antibacterial and antifungal references, respectively. Most of the newly synthesized compounds showed good antimicrobial activities with respect to the control drugs. The results of antimicrobial activities were shown in Table 2. Data showed that most of compounds have superior significant antifungal potency to antibacterial potency. Compound $\mathbf{8 c}$ exhibited the highest potency against all tested organisms with respect to reference drugs. Compounds $\mathbf{8 d}$ and $\mathbf{1 3 b}$ inhibited the growth of Staphylococcus aureus ATCC 29213 with inhibition zones 23, $22 \mathrm{~mm}$, respectively, while compound 5 showed excellent activities against Klebsiella pneumoniae ATCC13883; Pseudomonas aeruginosa ATCC27953; and E. coli ATCC 25922 with inhibition zone about $24 \mathrm{~mm}$. Also, compound $8 \mathrm{c}$ showed the highest activity against Staphylococcus aureus ATCC 29213, Saccharomyces cerevisiae, and Candida albicans NRRL Y-477 with inhibition zone about $23 \mathrm{~mm}$.
2.2.2. Minimum Inhibitory Concentration (MIC). The minimum inhibitory concentration (MIC) of the synthesized compounds against highly inhibited organisms is reported in Table 3. Compounds 5 revealed low MIC $(200 \mu \mathrm{g} / \mathrm{mL})$ against Staphylococcus aureus ATCC 29213, B. megaterium ATCC 9885, and Candida albicans NRRL Y-477, respectively. On the other hand, compound 8a exhibited high MIC $(16 \mu \mathrm{g} / \mathrm{mL})$ against B. subtilis ATCC6633 (Table 3).

\section{Experimental}

3.1. Chemistry. All melting points were taken on Electrothermal IA 9000 series digital melting point apparatus. Elemental analytical data were carried from the microanalytical unit, Cairo University, Giza, Egypt. The IR spectra were recorded in potassium bromide disks on a JASCO FT/IR-6100. ${ }^{1} \mathrm{H}$ NMR spectra were run on JOEL-ECA $500 \mathrm{MHz}$ in deuterateddimethyl sulphoxide (DMSO- $\left.\mathrm{d}_{6}\right)$. Chemical shifts values $(\delta)$ are given in parts per million (ppm). The mass spectra were performed using mass Varian MAT CH-5 spectrometer at 70 eV. (E)-1-(benzofuran-2-yl)-3-(4-fluorophenyl)prop-2-en1-one 3 [10]; hydrazonoyl halides [11]; 1-(benzofuran-2-yl)2-bromoethanone 9 [12]; 3-(2-bromoacetyl)-2H-chromen-2one 10 [13]; and 2,3-dichloroquinoxaline [14] were prepared according to the literature.

3.1.1. 3-(Benzofuran-2-yl)-5-(4-fluorophenyl)-4,5-dihydro-1Hpyrazole-1-carbothioamide (5). To a suspension of chalcone 3 (10 mmol, $2.66 \mathrm{~g})$ and sodium hydroxide ( $25 \mathrm{mmol}, 1.0 \mathrm{~g})$ in ethanol $(50 \mathrm{~mL})$, thiosemicarbazide $(12 \mathrm{mmol}, 1.1 \mathrm{~g})$ was added. The mixture was refluxed for $12 \mathrm{~h}$, then left to cool; the solid product was filtered off, washed with ethanol, and dried.

Yield 52\%; m.p. 260-2 ${ }^{\circ}$ C; IR (KBr) $\nu \max / \mathrm{cm}^{-1} 3460,3335$ $\left(\mathrm{NH}_{2}\right) ;{ }^{1} \mathrm{H}$ NMR $\left(\mathrm{DMSO}-\mathrm{d}_{6}\right) \delta 3.09,3.14(\mathrm{dd}, 1 \mathrm{H}, \mathrm{CH}, J=$ $3.05 \mathrm{~Hz}, J=3.05 \mathrm{~Hz}), 3.94,4.06(\mathrm{dd}, 1 \mathrm{H}, \mathrm{CH}, J=11.45 \mathrm{~Hz}, J=$ $11.5 \mathrm{~Hz}), 5.88(\mathrm{t}, 1 \mathrm{H}, \mathrm{CH}, J=3.05 \mathrm{~Hz}, J=7.65 \mathrm{~Hz}), 7.13-7.43(\mathrm{~m}$, 9H, Ar-H), 9.44 (s, 2H, $\mathrm{NH}_{2}, \mathrm{D}_{2} \mathrm{O}$-exchangeable); MS $\mathrm{m} / z$ (\%): $339\left(\mathrm{M}^{+}, 75\right), 60(100)$.

3.1.2. General Procedure for Compounds $8 \boldsymbol{8}-\boldsymbol{d}$; $\mathbf{1 3 a - d ; 1 4}$ 15; and 16. To a suspension of compound 5 ( $1 \mathrm{mmol}$, $0.34 \mathrm{~g})$ in ethanol $(20 \mathrm{~mL})$, the $1 \mathrm{mmol}$ of appropriate reagent \{(appropriate hydrozonoyl chlorides $6+\mathrm{Et}_{3} \mathrm{~N}$ ); (appropriate phenacyl bromides 9); (2-bromoacetylbenzofuran 10); (3bromoacetylcoumarin 11); or (3,4-dichloroquinoxaline 12)\} was added and heated under reflux for $4 \mathrm{~h}$. After cooling, the precipitate was collected by suction filtration.

3.1.3. 2-(3-(Benzofuran-2-yl)-5-(4-fluorophenyl)-4,5-dihydro1H-pyrazol-1-yl)-4-methyl-5-(phenyldiazenyl)thiazole (8a). Yield 58\%; m.p.180- $2{ }^{\circ} \mathrm{C}$; ${ }^{1} \mathrm{H}$ NMR (DMSO-d 6 ) $\delta 2.54$ (s, 3H, $\left.\mathrm{CH}_{3}\right), 4.09,4.11(2 \mathrm{dd}, 2 \mathrm{H}, \mathrm{CH}, J=10.7 \mathrm{~Hz}, J=9.95 \mathrm{~Hz}), 5.87$ $(\mathrm{t}, 1 \mathrm{H}, \mathrm{CH}, J=10.7 \mathrm{~Hz}, J=9.95 \mathrm{~Hz}), 7.18-7.73(\mathrm{~m}, 14 \mathrm{H}, \mathrm{Ar}-\mathrm{H})$; MS m/z (\%): $481\left(\mathrm{M}^{+}, 75\right), 95$ (100).

3.1.4. 2-(3-(Benzofuran-2-yl)-5-(4-fluorophenyl)-4,5-dihydro1H-pyrazol-1-yl)-5-((4-fluorophenyl)diazenyl)-4-methylthiazole (8b). Yield 66\%; m.p. 201- ${ }^{\circ} \mathrm{C} ;{ }^{1} \mathrm{H}$ NMR (DMSO- $\mathrm{d}_{6}$ ) $\delta 2.54\left(\mathrm{~s}, 3 \mathrm{H}, \mathrm{CH}_{3}\right), 4.07,4.12(2 \mathrm{dd}, 2 \mathrm{H}, \mathrm{CH}, J=10.7 \mathrm{~Hz}$, 
TABLE 2: Antimicrobial activity expressed as inhibition diameter zones in millimeters $(\mathrm{mm})$ of chemical compounds against the pathological strains based on well diffusion assay.

\begin{tabular}{|c|c|c|c|c|c|c|c|c|c|}
\hline \multirow[b]{2}{*}{$\begin{array}{l}\text { Chem. } \\
\text { compd. }\end{array}$} & \multicolumn{4}{|c|}{ Gram-positive bacteria } & \multicolumn{3}{|c|}{ Gram-negative bacteria } & \multicolumn{2}{|c|}{ Yeast } \\
\hline & $\begin{array}{c}\text { Staphylococcus } \\
\text { aureus } \\
\text { ATCC29213 }\end{array}$ & $\begin{array}{l}\text { B. subtilis } \\
\text { ATCC6633 }\end{array}$ & $\begin{array}{c}B . \\
\text { megaterium } \\
\text { ATCC9885 }\end{array}$ & $\begin{array}{c}\text { Sarcina } \\
\text { lutea }\end{array}$ & $\begin{array}{c}\text { Klebsiella } \\
\text { pneumoniae } \\
\text { ATCC13883 } \\
\end{array}$ & $\begin{array}{c}\text { Pseudomonas } \\
\text { aeruginosa } \\
\text { ATCC } 27953\end{array}$ & $\begin{array}{c}\text { E. coli } \\
\text { ATCC } 25922\end{array}$ & $\begin{array}{c}\text { Saccharomyces } \\
\text { cerevisiae }\end{array}$ & $\begin{array}{c}\text { Candida } \\
\text { albicans } \\
\text { NRRL Y-477 }\end{array}$ \\
\hline 5 & 18 & 17 & 20 & 23 & 24 & 23 & 24 & 22 & 18 \\
\hline $8 \mathbf{a}$ & 19 & 16 & 18 & 18 & 17 & 19 & 23 & 20 & 18 \\
\hline $8 b$ & 19 & 18 & 17 & 28 & 18 & 19 & 24 & 21 & 18 \\
\hline $8 c$ & 25 & 26 & 23 & 30 & 34 & 33 & 31 & 22 & 23 \\
\hline $8 d$ & 23 & 12 & 17 & 18 & 16 & 18 & 24 & 20 & 18 \\
\hline $13 a$ & 22 & 18 & 28 & 21 & 23 & 19 & 23 & 20 & 16 \\
\hline $13 b$ & 20 & 18 & 19 & 22 & 20 & 21 & 24 & 19 & 16 \\
\hline 14 & 19 & 18 & 20 & 23 & 22 & 22 & 25 & 24 & 20 \\
\hline 15 & 20 & 17 & 20 & 24 & 21 & 19 & 25 & 20 & 18 \\
\hline 16 & 19 & 16 & 21 & 20 & 21 & 20 & 27 & 21 & 16 \\
\hline Ciprofloxacin & 20 & 22 & 24 & 20 & 25 & 24 & 23 & N.A. & N.A. \\
\hline Ketoconazole & N.A. & N.A. & N.A. & N.A. & N.A. & N.A. & N.A. & 23 & 22 \\
\hline
\end{tabular}

The experiment was carried out in triplicate, and the average zone of inhibition was calculated; N.A. (no activity).

TABLE 3: Minimum inhibitory concentration $(\mu \mathrm{g} / \mathrm{mL})$ against the pathological strains based on twofold serial dilution technique.

\begin{tabular}{|c|c|c|c|c|c|c|c|c|c|}
\hline \multirow[b]{2}{*}{$\begin{array}{l}\text { Chem. } \\
\text { compds }\end{array}$} & \multicolumn{4}{|c|}{ Gram-positive bacteria } & \multicolumn{3}{|c|}{ Gram-negative bacteria } & \multicolumn{2}{|c|}{ Yeast } \\
\hline & $\begin{array}{c}\text { Staphylococcus } \\
\text { aureus } \\
\text { ATCC29213 }\end{array}$ & $\begin{array}{l}\text { B. subtilis } \\
\text { ATCC6633 }\end{array}$ & $\begin{array}{c}B . \\
\text { megaterium } \\
\text { ATCC } 9885\end{array}$ & $\begin{array}{c}\text { Sarcina } \\
\text { lutea }\end{array}$ & $\begin{array}{c}\text { Klebsiella } \\
\text { pneumoniae } \\
\text { ATCC13883 }\end{array}$ & $\begin{array}{c}\text { Pseudomonas } \\
\text { aeruginosa } \\
\text { ATCC } 27953\end{array}$ & $\begin{array}{c}\text { E. coli } \\
\text { ATCC } 25922\end{array}$ & $\begin{array}{c}\text { Saccharomyces } \\
\text { cerevisiae }\end{array}$ & $\begin{array}{c}\text { Candida } \\
\text { albicans } \\
\text { NRRL Y-477 }\end{array}$ \\
\hline 5 & 200 & - & 200 & 100 & 100 & 100 & 100 & 100 & 200 \\
\hline $8 a$ & 19 & 16 & 200 & 100 & - & 200 & 100 & 200 & 200 \\
\hline $8 b$ & 100 & 200 & - & 50 & 200 & 200 & 100 & 200 & - \\
\hline $8 c$ & 50 & 50 & 100 & 50 & 25 & 50 & 25 & 200 & 100 \\
\hline $8 d$ & 50 & - & - & 100 & - & 200 & 100 & 200 & 200 \\
\hline $13 \mathbf{a}$ & 200 & 200 & 200 & 100 & 200 & 200 & 100 & 200 & - \\
\hline $13 b$ & 100 & 200 & 50 & 100 & 100 & 200 & 100 & 200 & - \\
\hline 14 & 200 & 200 & 200 & 100 & 100 & 100 & 50 & 100 & 200 \\
\hline 15 & 200 & - & 200 & 100 & 100 & 200 & 50 & 200 & 200 \\
\hline 16 & 200 & - & 20 & 100 & 100 & 200 & 50 & 200 & - \\
\hline Ciprofloxacin & 25 & 25 & 25 & 25 & 25 & 25 & 25 & N.A. & N.A. \\
\hline Ketoconazole & N.A. & N.A. & N.A. & N.A. & N.A. & N.A. & N.A. & 25 & 25 \\
\hline
\end{tabular}

$J=9.95 \mathrm{~Hz}), 5.89(\mathrm{t}, 1 \mathrm{H}, \mathrm{CH}, J=10.7 \mathrm{~Hz}, J=9.95 \mathrm{~Hz})$, 7.19-7.73 (m, 13H, Ar-H); MS m/z (\%): $499\left(\mathrm{M}^{+}, 80\right), 95(100)$.

3.1.5. 2-(3-(Benzofuran-2-yl)-5-(4-fluorophenyl)-4,5-dihydro1H-pyrazol-1-yl)-5-((4-chlorophenyl)diazenyl)-4-methylthiazole (8c). Yield 72\%; m.p. 206-8 ${ }^{\circ} \mathrm{C} ;{ }^{1} \mathrm{H}$ NMR (DMSO-d 6 ) $\delta$ $2.53\left(\mathrm{~s}, 3 \mathrm{H}, \mathrm{CH}_{3}\right), 4.10,4.34(2 \mathrm{dd}, 2 \mathrm{H}, \mathrm{CH}, J=10.7 \mathrm{~Hz}, J=$ $9.95 \mathrm{~Hz}), 5.89$ (t, $1 \mathrm{H}, \mathrm{CH}, J=10.7 \mathrm{~Hz}, J=9.95 \mathrm{~Hz}), 7.19-7.73$ (m, 13H, Ar-H); MS m/z (\%): $515\left(\mathrm{M}^{+}, 70\right), 95$ (100).

3.1.6. 2-(3-(Benzofuran-2-yl)-5-(4-fluorophenyl)-4,5-dihydro1H-pyrazol-1-yl)-5-((4-bromophenyl)diazenyl)-4-methylthiazole $(\mathbf{8 d})$. Yield 75\%; m.p. 128-30 ${ }^{\circ}$; ${ }^{1} \mathrm{H}$ NMR (DMSO- $\mathrm{d}_{6}$ ) $\delta 2.55\left(\mathrm{~s}, 3 \mathrm{H}, \mathrm{CH}_{3}\right), 4.10,4.34(2 \mathrm{dd}, 2 \mathrm{H}, \mathrm{CH}, J=10.7 \mathrm{~Hz}$,
$J=9.95 \mathrm{~Hz}), 5.87(\mathrm{t}, 1 \mathrm{H}, \mathrm{CH}, J=10.7 \mathrm{~Hz}, J=9.95 \mathrm{~Hz})$, 7.19-7.72 (m, 13H, Ar-H); MS m/z (\%): $561\left(\mathrm{M}^{+}, 62\right), 95$ (100).

3.1.7. 2-(3-(Benzofuran-2-yl)-5-(4-fluorophenyl)-4,5-dihydro1H-pyrazol-1-yl)-4-(4-chlorophenyl)thiazole (13a). Yield 49\%; m.p. 176-8 ${ }^{\circ}$; ${ }^{1} \mathrm{H}$ NMR (DMSO-d 6 ) $\delta$ 4.01-4.05 (dd, $\left.2 \mathrm{H}, \mathrm{CH}_{2}, J=11.45 \mathrm{~Hz}, J=10.33 \mathrm{~Hz}\right), 5.73(\mathrm{t}, 1 \mathrm{H}, \mathrm{CH}, J=$ $5.35 \mathrm{~Hz}, J=6.1 \mathrm{~Hz}), 7.17-7.92(\mathrm{~m}, 14 \mathrm{H}, \mathrm{Ar}-\mathrm{H}) ; \mathrm{MS} m / z(\%)$ : $473\left(\mathrm{M}^{+}, 80\right), 91(100)$.

3.1.8. 2-(3-(Benzofuran-2-yl)-5-(4-fluorophenyl)-4,5-dihydro1H-pyrazol-1-yl)-4-(4-bromophenyl)thiazole (13b). Yield 58\%; m.p. $208-10^{\circ} \mathrm{C}$; ${ }^{1} \mathrm{H}$ NMR (DMSO-d 6 ) $\delta$ 4.01-4.08 $\left(\mathrm{dd}, 2 \mathrm{H}, \mathrm{CH}_{2}, J=11.45 \mathrm{~Hz}, J=10.33 \mathrm{~Hz}\right), 5.71(\mathrm{t}, 1 \mathrm{H}, \mathrm{CH}$, 
$J=5.35 \mathrm{~Hz}, J=6.1 \mathrm{~Hz}), 7.17-7.92(\mathrm{~m}, 14 \mathrm{H}, \mathrm{Ar}-\mathrm{H}) ; \mathrm{MS} m / z$ (\%): $518\left(\mathrm{M}^{+}, 49\right), 91(100)$.

3.1.9. 4-(Benzofuran-2-yl)-2-(3-(benzofuran-2-yl)-5-(4-fluorophenyl)-4,5-dihydro-1H-pyrazol-1-yl)thiazole (14). Yield 63\%; m.p. 252-4 ${ }^{\circ} \mathrm{C} ;{ }^{1} \mathrm{H}$ NMR (DMSO-d 6 ) $\delta$ 4.04-4.07 (dd, $\left.2 \mathrm{H}, \mathrm{CH}_{2}, J=11.45 \mathrm{~Hz}, J=10.33 \mathrm{~Hz}\right), 5.73(\mathrm{t}, 1 \mathrm{H}, \mathrm{CH}, J=$ $5.35 \mathrm{~Hz}, J=6.1 \mathrm{~Hz}), 6.88$ (s, 2H, benzofuryl-H), 7.19-7.70 (m, $13 \mathrm{H}, \mathrm{Ar}-\mathrm{H})$; $\mathrm{MS} \mathrm{m} / z$ (\%): $479\left(\mathrm{M}^{+}, 100\right)$.

3.1.10. 3-(2-(3-(Benzofuran-2-yl)-5-(4-fluorophenyl)-4,5-dihydro-1H-pyrazol-1-yl)thiazol-4-yl)-2H-chromen-2-one (15). Yield 74\%; m.p. 233- $4^{\circ} \mathrm{C} ;{ }^{1} \mathrm{H}$ NMR (DMSO-d 6 ) $\delta 4.05,4.08$ $\left(\mathrm{dd}, 2 \mathrm{H}, \mathrm{CH}_{2}, J=11.45 \mathrm{~Hz}, J=10.33 \mathrm{~Hz}\right), 5.72(\mathrm{t}, 1 \mathrm{H}, \mathrm{CH}, J=$ $5.35 \mathrm{~Hz}, J=6.1 \mathrm{~Hz}), 7.22-7.72(\mathrm{~m}, 14 \mathrm{H}, \operatorname{Ar}-\mathrm{H}), 8.23$ (s, $1 \mathrm{H}$, coumarinyl-H); MS m/z (\%): $507\left(\mathrm{M}^{+}, 100\right)$.

3.1.11. 2-(3-(Benzofuran-2-yl)-5-(4-fluorophenyl)-4,5-dihydro1H-pyrazol-1-yl)thiazolo[5,4-b]quinoxaline (16). Yield 74\%; m.p. $256-8^{\circ} \mathrm{C} ;{ }^{1} \mathrm{H}$ NMR (DMSO-d 6 ) $\delta 4.02,4.06$ (dd, $2 \mathrm{H}$, $\left.\mathrm{CH}_{2}, J=11.45 \mathrm{~Hz}, J=10.33 \mathrm{~Hz}\right), 5.70(\mathrm{t}, 1 \mathrm{H}, \mathrm{CH}, J=5.35 \mathrm{~Hz}$, $J=6.1 \mathrm{~Hz}), 7.22-7.72(\mathrm{~m}, 13 \mathrm{H}, \mathrm{Ar}-\mathrm{H})$; MS m/z (\%): $465\left(\mathrm{M}^{+}\right.$, 100).

3.2. Antimicrobial Activity. Chemical compounds were individually tested against a panel of Gram-positive and Gramnegative bacterial pathogens and yeast. Antimicrobial tests were carried out by the agar well diffusion method [15] using $100 \mu \mathrm{L}$ of suspension containing $1 \times 10^{8} \mathrm{CFU} / \mathrm{mL}$ of pathological tested bacteria and $1 \times 10^{6} \mathrm{CFU} / \mathrm{mL}$ of yeast spread on nutrient agar (NA) and Sabourund dextrose agar (SDA), respectively. After the media had cooled and solidified, wells (10 mm in diameter) were made in the solidified agar and loaded with $100 \mu \mathrm{L}$ of tested compound solution prepared by dissolving $100 \mathrm{mg}$ of the chemical compound in one $\mathrm{mL}$ of dimethyl sulfoxide (DMSO). The inculcated plates were then incubated for $24 \mathrm{~h}$ at $37^{\circ} \mathrm{C}$ for bacteria and at $28^{\circ} \mathrm{C}$ for yeast. Negative controls were prepared using DMSO employed for dissolving the tested compound. Ciprofloxacin $(50 \mu \mathrm{g} / \mathrm{mL})$ and ketoconazole $(50 \mu \mathrm{g} / \mathrm{mL})$ were used as standard for antibacterial and antifungal activities respectively. After incubation time, antimicrobial activity was evaluated by measuring the zone of inhibition against the test organisms and compared with that of the standard. The observed zone of inhibition is presented in Table 2. Antimicrobial activities were expressed as inhibition diameter zones in millimeters $(\mathrm{mm})$. The experiment was carried out in triplicate, and the average zone of inhibition was calculated.

\subsection{Minimal Inhibitory Concentration (MIC) Measurement.} The bacteriostatic activity of the active compounds (having inhibition zones (IZ) $\geq 18 \mathrm{~mm}$ ) was then evaluated using the twofold serial dilution technique [16]. Twofold serial dilutions of the tested compounds solutions were prepared using the proper nutrient broth. The final concentration of the solutions was $200,100,50$, and $25 \mu \mathrm{g} / \mathrm{mL}$. The tubes were then inoculated with the test organisms, grown in their suitable broth at $37^{\circ} \mathrm{C}$ for 24 hours for bacteria (about $1 \times$ $10^{8} \mathrm{CFU} / \mathrm{mL}$ and $1 \times 10^{6} \mathrm{CFU} / \mathrm{mL}$ of yeast), and each $5 \mathrm{~mL}$ received $0.1 \mathrm{~mL}$ of the above inoculum and incubated at $37^{\circ} \mathrm{C}$ for 24 hours. The lowest concentration showing no growth was taken as the minimum inhibitory concentration (MIC).

\section{Conclusion}

Novel pyrazolylthiazoles, with potential antimicrobial activity, were prepared from available 2-acetylbenzofuran. Firstly, chalcone 3 was obtained by condensation with 4 -fluorobenzaldehyde in alcoholic $\mathrm{NaOH}$. This precursor reacted with thiosemicarbazide in strong basic medium to afford the $N$ pyrazoline thioamide 5. Reaction of the latter with different haloketones and 2,3-dichloroquinoxaline gave the target pyrzolinylthiazoles 8-16. The new compounds were tested for their antimicrobial activities and significant activities due to presence of three nucleuses: benzofuran, pyrazole, and thiazole. Also, some substituent increases the antimicrobial activities such as chloro substituent in compounds $\mathbf{8 c}$ and 13a.

\section{References}

[1] H. Patel, V. Ugale, A. Ingale, and S. Bari, "Synthesis and antimicrobial evaluation of pyrazo-thiazoles," Letters in Drug Design \& Discovery, vol. 9, pp. 840-847, 2012.

[2] C.-Y. Zhang, X.-H. Liu, B.-L. Wang, S.-H. Wang, and Z.-M. Li, "Synthesis and antifungal activities of new pyrazole derivatives via 1,3-dipolar cycloaddition reaction," Chemical Biology and Drug Design, vol. 75, no. 5, pp. 489-493, 2010.

[3] T. D. Sherman, M. V. Duke, R. D. Clark, E. F. Sanders, H. Matsumoto, and S. O. Duke, "Pyrazole phenyl ether herbicides inhibit protoporphyrinogen oxidase," Pesticide Biochemistry and Physiology, vol. 40, no. 3, pp. 236-245, 1991.

[4] H. Song, Y. Liu, L. Xiong, Y. Li, N. Yang, and Q. Wang, "Design, synthesis, and insecticidal activity of novel pyrazole derivatives containing $\alpha$-hydroxymethyl-N-benzyl carboxamide, $\alpha$ chloromethyl-N-benzyl carboxamide, and 4,5-dihydrooxazole moieties," Journal of Agricultural and Food Chemistry, vol. 60, no. 6, pp. 1470-1479, 2012.

[5] B. F. Abdel-Wahab, A. Sediek, H. A. Mohamed, and G. E. A. Awad, "Novel 2-pyrazolin-1-ylthiazoles as potential antimicrobial agents," Letters in Drug Design \& Discovery, vol. 10, no. 2, pp. 111-118, 2013.

[6] B. F. Abdel-Wahab, E. Abdel-Latif, H. A. Mohamed, and G. E. A. Awad, "Design and synthesis of new 4-pyrazolin-3-yl-1,2,3-triazoles and 1,2,3-triazol-4-yl-pyrazolin-1-ylthiazoles as potential antimicrobial agents," European Journal of Medicinal Chemistry, vol. 52, pp. 263-268, 2012.

[7] B. F. Abdel-Wahab, R. E. Khidre, and G. E. A. Awad, "Regioselective synthesis and antimicrobial activities of some novel aryloxyacetic acid derivatives," European Journal of Medicinal Chemistry, vol. 50, pp. 55-62, 2012.

[8] B. F. Abdel-Wahab, H. A. Mohamed, A. A. Farahat, and K. M. Dawood, "Synthetic accesses to azolylthiazoles," Heterocycles, vol. 83, no. 12, pp. 2731-2767, 2011.

[9] R. E. Khidre, B. F. Abdel-Wahab, and F. A.-R. Badria, "New quinoline-based compounds for analgesic and anti-inflammatory evaluation," Letters in Drug Design \& Discovery, vol. 8, no. 7, pp. 640-648, 2011.

[10] P. H. Richter, M. Elsner, and B. Vogt, "Preparation of benzo[b] furan-derived ketone amidinohydrazone class-III antiarrhythmic agents," European Patent Applications, EP 778274, 1997. 
[11] L. Czollner, G. Szilagyi, J. Lango, and J. J. Janaky j., "Synthesis of new 1,5-diphenyl-3-1H-1,2,4-triazoles substituted with $H$-,alkyl, or carboxyl groups at C-3," Archiv der Pharmazie, vol. 323, no. 4, pp. 225-227, 1990.

[12] R. L. Shriner and J. Anderson, "Derivatives of coumaran. VI. Reduction of 2-acetobenzofuran and its derivatives," Journal of the American Chemical Society, vol. 61, no. 10, pp. 2705-2708, 1939.

[13] C. F. Koelsch, "Bromination of 3-acetocoumarin," Journal of the American Chemical Society, vol. 72, no. 7, pp. 2993-2995, 1950.

[14] V. S. H. Krishnan, K. S. Chowdary, and P. K. Dubey, "Studies in the syntheses of s-triazolo[4,3-a] quinoxalines," Indian Journal of Chemistry B, vol. 38, no. 1, pp. 45-51, 1999.

[15] C. Perez, M. Pauli, and P. Bazevque, "An antibiotic assay by the agar well diffusion method," Acta Biologica et Medica Experimentalis, vol. 15, pp. 113-115, 1990.

[16] A. C. Scott, "Laboratory control of antimicrobial therapy," in Mackie and MacCartney Practical Medical Microbiology, J. G. Collee, J. P. Duguid, A. G. Fraser, and B. P. Marmion, Eds., vol. 2, pp. 161-181, Churchill Livingstone, Edinburgh, Scotland, 13th edition, 1989. 

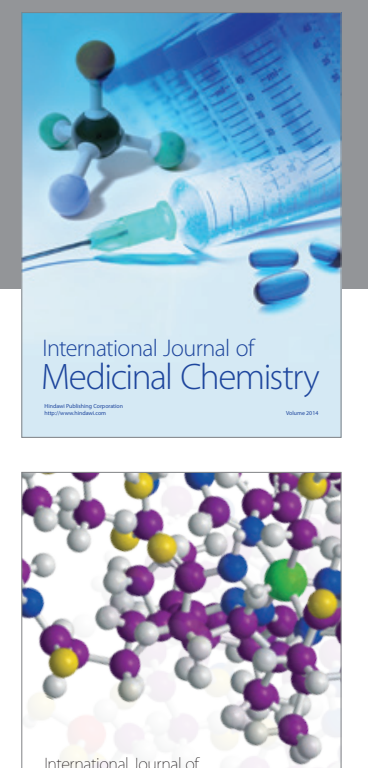

\section{Carbohydrate} Chemistry

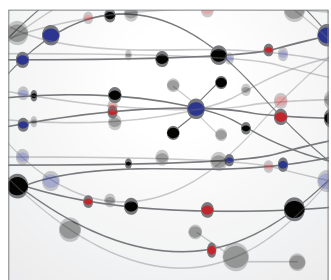

The Scientific World Journal
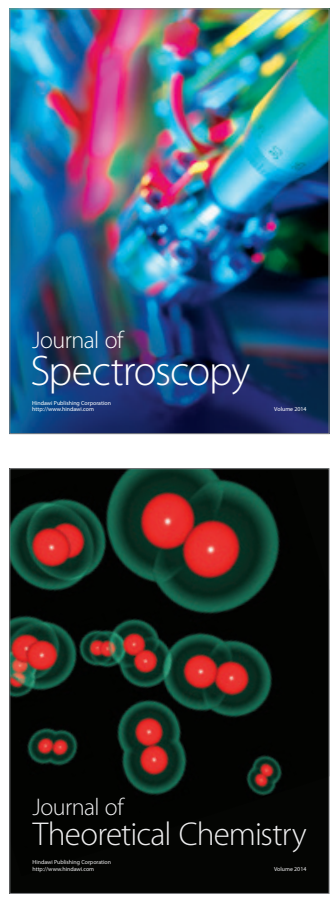
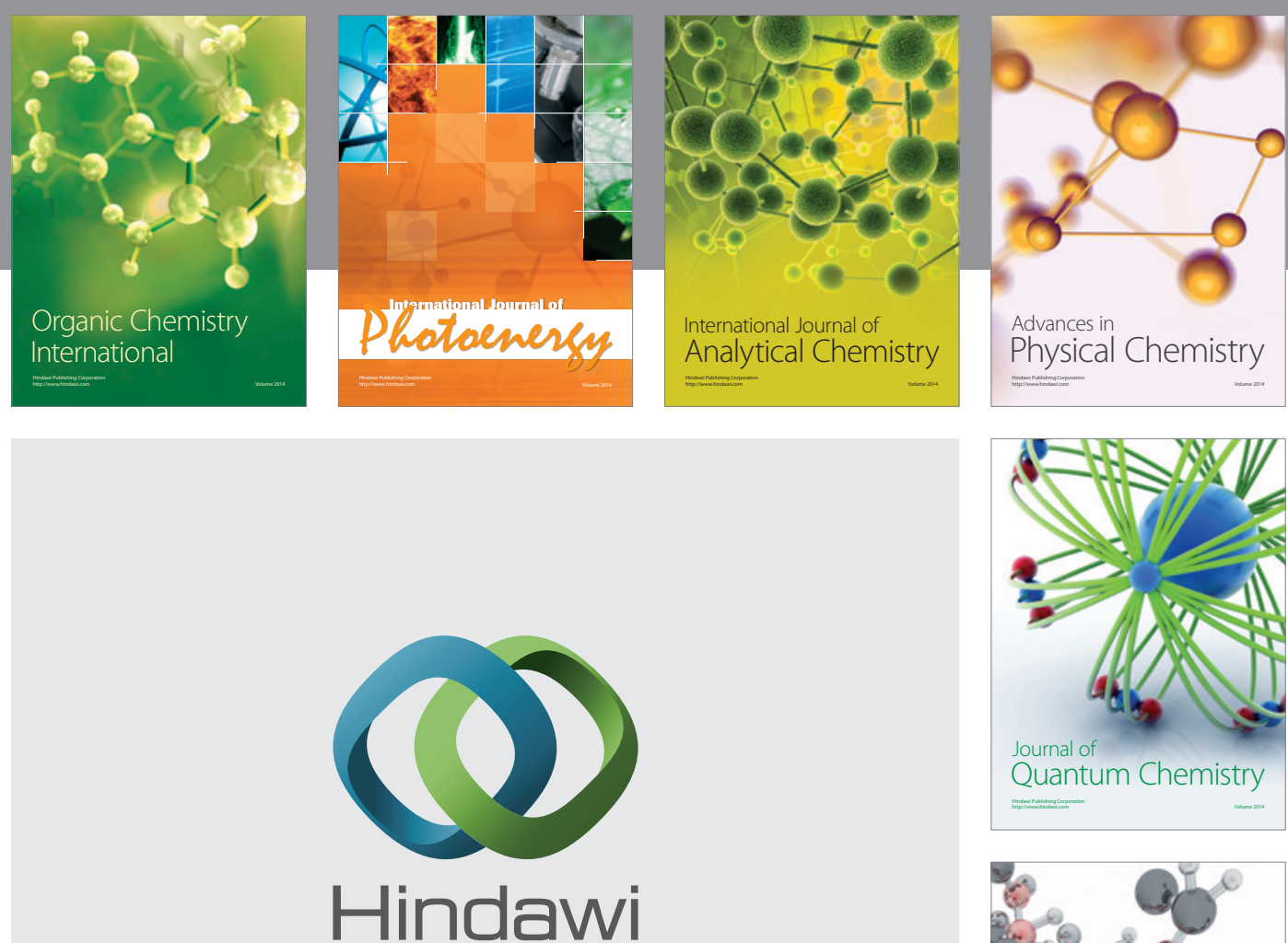

Submit your manuscripts at

http://www.hindawi.com

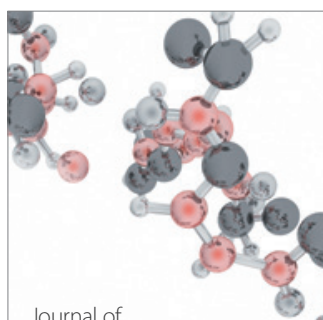

Analytical Methods

in Chemistry

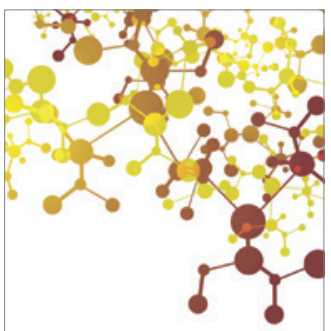

Journal of

Applied Chemistry

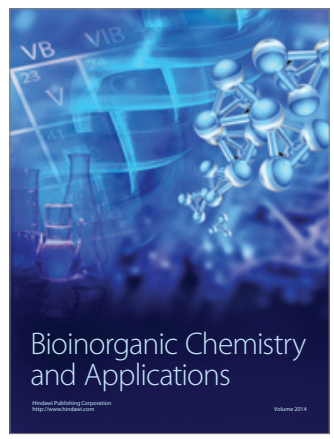

Inorganic Chemistry
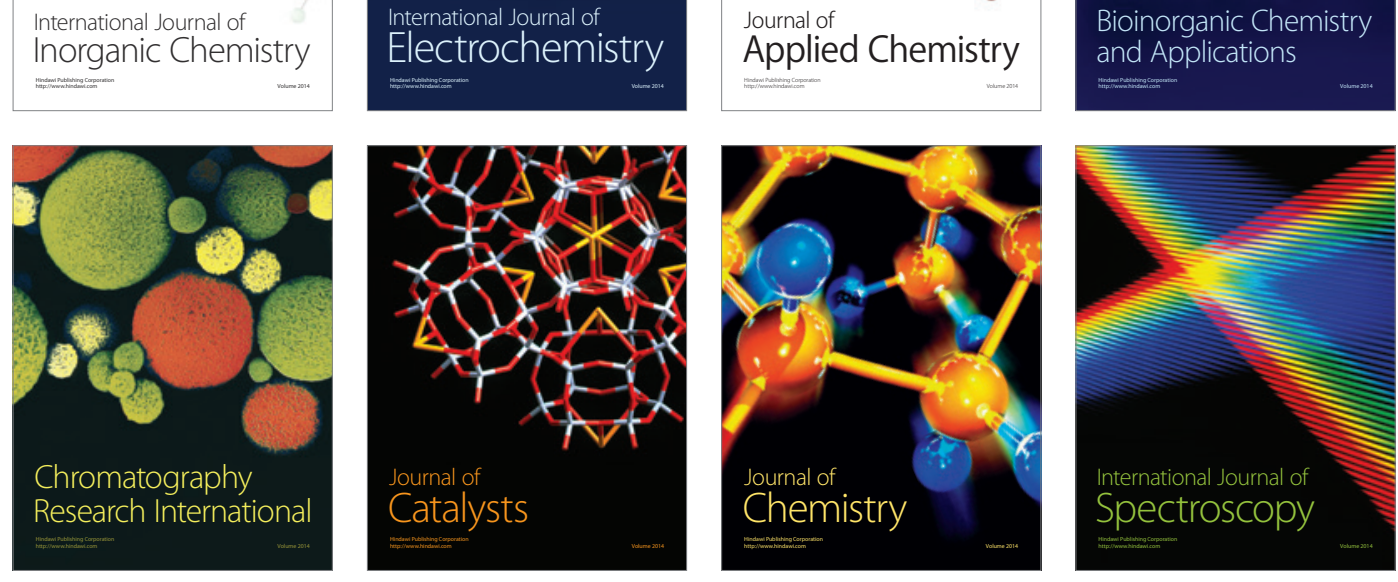